\title{
Elevated basal cytosolic calcium of endothelial cells influences the post-surgical outcome in diabetic CTS
}

\author{
IM Bin-Jaliah ${ }^{1}$, MEA Shariff ${ }^{1,2}$, PS Nayar², HC Chandramoorthy ${ }^{3}$ \\ ${ }^{1}$ Department of Physiology, College of Medicine, King Khalid University, Abha, Saudi Arabia \\ ${ }^{2}$ Department of Orthopedics, Jubilee Mission Medical College, Thrissur, Kerala, India \\ ${ }^{3}$ Department of Microbiology and Parasitology, College of Medicine, King Khalid University, Abha, Saudi Arabia
}

Received: January 20, 2016

Accepted: September 19, 2016

\begin{abstract}
Background: Type 2 diabetes mellitus (T2DM)-induced neuropathy and ischemia-reperfusion post-surgery prolong carpal tunnel syndrome (CTS) pathology, but the effect of T2DM on the prognostic outcome of carpal tunnel (CT) release surgery needs to be investigated. Materials and methods: A total of 64 individuals with CTS underwent $\mathrm{CT}$ release surgery. HbAlc levels identified their diabetic status. The individual prognostic outcomes were measured by nerve conduction velocity (NCV), amplitude, and latency. Measurement of $\left[\mathrm{Ca}^{2+}\right]_{\mathrm{c}}$ and reactive oxygen species (ROS) from isolated endothelial cells (ECs) revealed the oxidative burden of the normal and diabetic CTS phenotypes. Results: CTS individuals with HbAlc $>7$ showed decreased NCV $(\approx 22 \mathrm{~m} / \mathrm{s})$ and amplitude $(\approx 4.2 \mathrm{mV})$ with increased latency $(\approx 6 \mathrm{~ms})$, compared to groups with HbA1c $\leq 7$. Further to CT release surgery, the reversal of the nerve conduction to normalcy was greatly influenced by the diabetic profile of the individuals. Our results showed elevated basal $\left[\mathrm{Ca}^{2+}\right]_{\mathrm{c}}$ and corresponding high cytosolic ROS in the ECs isolated from individuals with $\mathrm{HbAlc}>7$ compared to the diabetic and healthy control groups. Conclusion: The individuals with diabetic index showed suboptimal neuronal performance pre- and post-CT release surgery. Oxidative stress mediated by high $\left[\mathrm{Ca}^{2+}\right]_{\mathrm{c}}$ and ROS of ECs dissipates to adjoining cells worsening the pathology of the untreated CTS.
\end{abstract}

Keywords: carpal tunnel syndrome, diabetic CTS, endothelial calcium, endothelial ROS, post surgery prolonged CTS, oxidative stress, T2DM complications

\section{Introduction}

Carpal tunnel syndrome (CTS) is the most frequently encountered entrapment neuropathy. The pathophysiological mechanism behind CTS is elevated carpal tunnel (CT) pressure due to repetitive strain injury caused by the compression of the median nerve, narrowing the tunnel space $(1,4,24)$. There are a few studies that have implicated less infiltration of the inflammatory cells. It may be noted that many studies have been directed toward pain management and post-operative surgical management strategies, such as oscillatory biofield therapy or endoscopy over conventional surgical measures (22). Apart from the idiopathic CTS, many metabolic syndromes have been attributed to be one of the factors for development of CTS with oxidative stress as a predisposing factor $(16,23)$. Diabetes has been linked with progression of CTS with classical presentation of neovascularization in subsynovial connective tissue increasing its vascular permeability (5). Although there is no single

Corresponding author: Dr. Harish C Chandramoorthy, PhD, FICS

Department of Microbiology and Parasitology, College of Medicine, King Khalid University

P.O. Box 641, Abha 61421, Saudi Arabia

Phone: +966 172418131; Fax: +966 172418067; E-mail: ccharishjabali@gmail.com 
predisposing factor likely to cause CTS, some studies have identified type 2 diabetes mellitus (T2DM) a major factor, complicating CTS progression as well as prognosis. About 10 times, more individuals with proven diabetic neuropathy had been diagnosed with CTS compared to $3 \%$ reported CTS from the general population (31). It is generally assumed that CTS is more likely to be associated with metabolic conditions, such as diabetes, obesity, osteoarthritis, and hypothyroidism. Many acquired conditions, such as smoking, alcohol abuse, and mental stress, have also been attributed to a higher incidence of CTS. None of the studies have identified the cellular pathology, which accompanies CTS due to the reduction of intraneural blood flow, edema formation, and endoneural hypoxia $(14,15,20,27)$.

One of the causes of CTS clinical manifestation is the presence of neural and synovial ischemia, which results in inflammatory cytokine infiltration at the tenosynovium. The exact mechanism behind the pro-inflammatory cytokine infiltration or ischemia is not clear $(9,12)$. However, none of the above investigations have reported on the activation of endothelial cells (ECs), which are the first line of cells to encounter cellular hypoxia due to increased interstitial pressure as a result of CTS $(21,30)$. Furthermore, there is no clear-cut report on the effects of the diabetes and its control on CTS or post-surgical outcome and management of CTS.

In the current investigation, we hypothesized that the reperfusion injury (post-surgery) and long term complicating T2DM induce huge oxidative stress via an increased intracellular calcium concentration $\left[\mathrm{Ca}^{2+}\right]_{c}$, which in turn triggers reactive oxygen species (ROS), skewing the cells toward death (19). The above sequence of altered $\left[\mathrm{Ca}^{2+}\right]_{\mathrm{c}}$ homeostasis may result in cellular pathology leading to the increased sensitivity of the nerve endings due the loss of protective endothelium exposing the base membrane. This then contributes more stimuli and thereby pain. Preliminary results of this study were presented at the 38th annual meeting of the Japan Neuroscience Society, Kobe, Japan, in 2015 (2).

\section{Materials and Methods}

\section{Subjects}

This study was undertaken in the Department of Orthopedics, Jubilee Mission Medical College, Thrissur, Kerala, South India, following approval from the Research and Ethical Committee (ethical clearance letter \#032008). A part of the work was done at the College of Medicine, King Khalid University, Abha, Saudi Arabia. All the individuals for the study were enrolled after obtaining their written consent. Individuals with uncontrolled diabetes were treated before surgery. Based on the HbA1c levels, the individuals were grouped into a non-diabetic control group with $\mathrm{HbA} 1 \mathrm{c}<5$, a controlled diabetic group with $\mathrm{HbA1c}=5-7$, and an uncontrolled diabetic group with $\mathrm{HbA1c}>7$. Data on clinical features, predisposing factors, and investigation including electrodiagnostic evaluation and physical examination were obtained from the case records. Diagnostic criteria for pre-operative evaluation were based on the standard American Academy of Neurology $(1,12)$.

\section{Glycated hemoglobin HbAlc}

The HbA1c levels were determined by the borate affinity assay (NycoCard, Axis-Shield PoC AS, Norway). Briefly, $2 \mathrm{ml}$ of blood was collected in an ethylenediamine tetraacetic acid tube, and HbAlc levels were assayed as per the instructions supplied with the kit (13). 
Nerve conduction studies

The neurophysiological measurements were performed in a warm room with the participants in a supine position. Nerve conduction velocity data were recorded using an RMS EMG EP Mark II instrument (Recorders and Medicare System, Chennai, India).

Median nerve conduction study. Silver surface electrodes were used; the recording electrodes were placed over the belly of the abductor pollicis brevis muscle and the reference electrodes were placed over the first metacarpophalangeal joint. The stimulation site for the wrist was the middle of the wrist between the tendons of the flexor carpi radialis and palmaris longus muscles and for the antecubital fossa medial to the biceps brachii muscle tendon with distal distance of $8 \mathrm{~cm}$; the ground electrodes were placed on the back of the hand. For the median sensory study, the recording electrodes were placed over the metacarpophalangeal joint of the index finger and the reference electrodes were placed 3-4 cm distally. The stimulation site for the wrist was the middle of the wrist between the tendons of the flexor carpi radialis and palmaris longus muscles with a distal distance of $14 \mathrm{~cm}$. The ground electrodes were placed on the back of the hand. Ulnar nerve conduction study. The participants were seated with their forearms partially flexed. Stimulating electrodes were placed at $5 \mathrm{~cm}$ below the medial epicondyle and at $5 \mathrm{~cm}$ above the medial epicondyle; the recording electrodes were placed over the abductor digiti minimi muscle on the ulnar side of the hand, i.e., between the fifth metacarpophalangeal joint and the pisiform bone. The compound muscle action potentials (CMAPs) were evoked by three electrical stimulations ( $0.1 \mathrm{~ms}$ duration, constant current pulse) of the ulnar nerve, starting with a minimum and progressing to the maximum intensity of the stimuli. Latency, amplitude, and nerve conduction velocity were assessed (1).

\section{EC isolation}

Five specimens of arteries and veins from each of the groups were obtained from individuals undergoing CTS surgery. The tissues were chilled and transported to the laboratory suspended in EC transport medium. Phosphate-buffered saline containing $100 \mathrm{U} / \mathrm{ml}$ penicillin and $100 \mu \mathrm{g} / \mathrm{ml}$ streptomycin was used to initially clean the tissues and the fibrous portion was scraped from the veins and arteries. The tissue was cut into $0.5 \mathrm{~cm} \times 0.5 \mathrm{~cm}$ squares approximately and flipped over in the tissue culture dish containing EC growth medium just bathing the tissues. The plates were incubated at $37{ }^{\circ} \mathrm{C}, 5 \% \mathrm{CO}_{2}$ for 2 weeks. Post first week, the tissue pieces were removed leaving the individual colonies to develop into a confluent monolayer. Phenotyping of ECs was carried out according to the standard protocol (19).

\section{Measurement of ROS}

Briefly, $10^{5}$ ECs were stained with $2^{\prime}$, $7^{\prime}$-dichlorofluorescein diacetate (H2DCF-DA; Molecular Probes Cat \# D399, Thermo Fisher Scientific, USA; $10 \mu \mathrm{M}$, an oxidation-sensitive dye) according to manufacturer's instructions. The cells were acquired immediately and analyzed using a BC Novus flow cytometer (8).

Measure of cytosolic calcium $\left[\mathrm{Ca}^{2+}\right]_{c}$

Intracellular calcium was assessed by Fluo-4 NW calcium Assay Kits (Molecular Probes Cat \# F36206, Thermo Fisher Scientific, USA). Briefly, ECs were grown on 96-well tissue culture plates, on the day of experiment. The growth medium is removed and $100 \mu \mathrm{l}$ of loading dye is added and incubated at $37^{\circ} \mathrm{C}$ for $45 \mathrm{~min}$. The plates were measured with the spectrometer on a setting of $494 \mathrm{~nm}$ excitation and $516 \mathrm{~nm}$ emission (3). The experiments were performed in triplicate. The data were analyzed and plotted using GraphPad Prism 5 software. 
Statistical analysis

Most of the patient data are expressed in real numbers or in percentage. Unless specified otherwise, all the laboratory experimental data were performed in triplicate at different time periods. The data are expressed as mean $\pm \mathrm{SE}$, and statistical significance was evaluated via one-way ANOVA and Student's $t$-test. A $P$ value of $<0.05$ was considered statistically significant. Data were plotted with GraphPad Prism v5.0 (GraphPad Co. Ltd., San Diego, USA).

\section{Results}

In this study, it was found that females were more affected by CTS compared with the males (Fig. 1a). The average female ages were lower (younger) compared with the male population (Fig. 1b). The CTS cases were more common on the right hand than the left hand or bilateral manifestations (Fig. 1c). The CTS individual's demographic data showed the clinical severity; pre- and post-surgery, irrespective of their diabetic complications and management (Table I). In this study, the CTS cases were segregated based on their HbAlc levels (Table II).

(a)

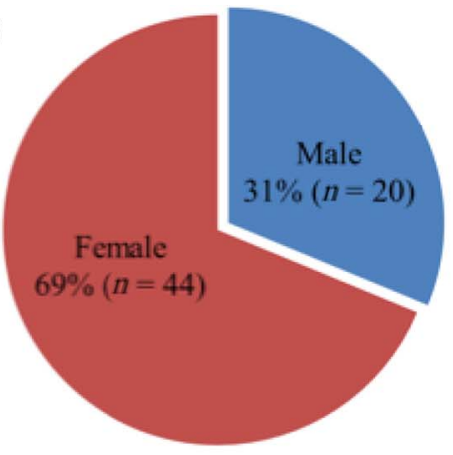

(c)

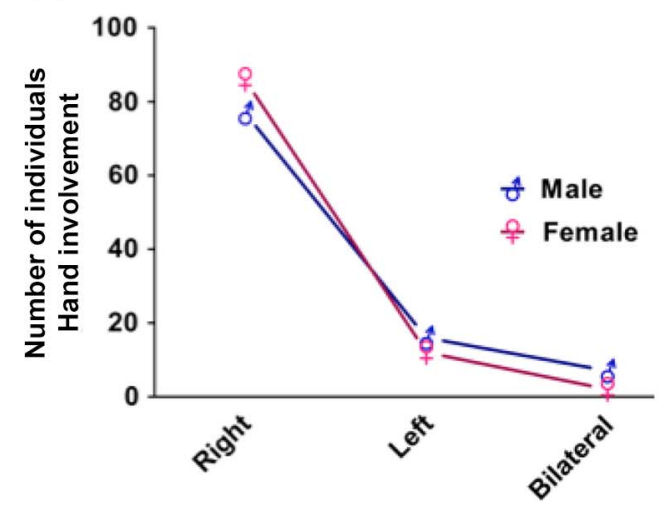

(b)

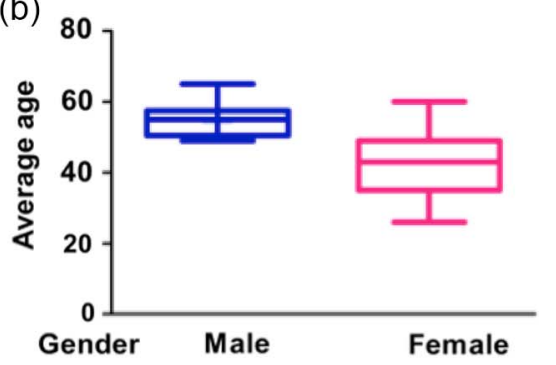

Fig. 1. CTS individuals' demographics. (a) Distribution of the male and female subjects with the CTS admitted in the study; male $(n=20)$ and female $(n=44)$. (b) Average age group in which CTS is more often reported.

Comparatively, younger females are more affected than the male population. (c) Involvement of hand in the CTS was uniform in the male and female subjects. This study showed that right-hand involvement was more prominent than the left- and bilateral-hand involvement 
Table I. Prominent clinical features of CTS and their recovery after surgical intervention

\begin{tabular}{|c|l|c|c|}
\hline \multirow{2}{*}{ Condition } & \multicolumn{1}{|c|}{ Symptoms } & $\begin{array}{c}\text { Pre-surgical observation, \% } \\
(\boldsymbol{n}=\mathbf{6 4})\end{array}$ & $\begin{array}{c}\text { Post-surgical recovery, \% } \\
(\boldsymbol{n}=\mathbf{6 4})\end{array}$ \\
\hline \multirow{5}{*}{ CTS } & Pain & 77 & 94 \\
\cline { 2 - 4 } & Paresthesia and/or numbness & 87 & 67 \\
\cline { 2 - 4 } & Muscle weakness & 66 & 86 \\
\cline { 2 - 4 } & Reflex sympathetic dystrophy & 57 & Neg \\
\cline { 2 - 4 } & Phalen's sign & 59 & Neg \\
\cline { 2 - 4 } & Tinel's sign & 50 & Neg \\
\hline
\end{tabular}

Table II. Type 2 diabetic profile

\begin{tabular}{|c|c|c|}
\hline S. No. & Diabetic profile $($ HbA1c) & Number of individuals $(\boldsymbol{n}=\mathbf{6 4})$ \\
\hline 1 & $\mathrm{HbA} 1 \mathrm{c}<5$ & 14 \\
\hline 2 & $\mathrm{HbA} 1 \mathrm{c}=5-7$ & 27 \\
\hline 3 & $\mathrm{HbA} 1 \mathrm{c}>7$ & 23 \\
\hline
\end{tabular}

Furthermore, this study did not alter the proper diabetic or post-surgical management of the individuals undergoing surgery. Appropriate pre-surgical care was taken for all the individuals; therefore, many of the diabetics were put on diabetic care management before the surgery to avoid diabetic complications, such as delayed wound healing. Table II shows the average $\mathrm{HbA} 1 \mathrm{c}$ levels and, for the sake of convenience, groups will be identified hereafter as Group I (controls, HbAlc $<5$; no diabetes), Group II (CTS with long controlled diabetes, HbAlc = 5-7), and Group III (CTS with uncontrolled diabetes/not known, HbAlc > 7). The values for the diabetic group with CTS are from pre-diabetic management before the surgery to demark the cases for its neuropathy as one of the standard and known complication of CTS due to T2DM. All the cases following the diabetic care management schedule showed good glycemic control.

\section{CTS characterized by altered nerve conduction}

Although for a brief period of time Group III with CTS had good glycemic control, the nerve conduction velocity (NCV) was very low compared with those of Groups I and II (Fig. 2a). The distal motor latency was prolonged and very high in Group III compared with Groups I and II (Fig. 2b). The amplitude was very much lower in Group III than in the other two groups and had a direct correlation with increasing HbA1c levels (Fig. 2c). Some of the patients did not follow up on treatment as their symptoms disappeared, and the data given are from the first few weeks of prognosis. Few individuals who had come for follow-up for nerve conduction studies initially showed Groups I and II having a clear improvement compared with those of Group III (Fig. 2a-c). The results clearly showed a decrease in the total latency and the increase of amplitude with increased restoration of normalcy. 
(a)

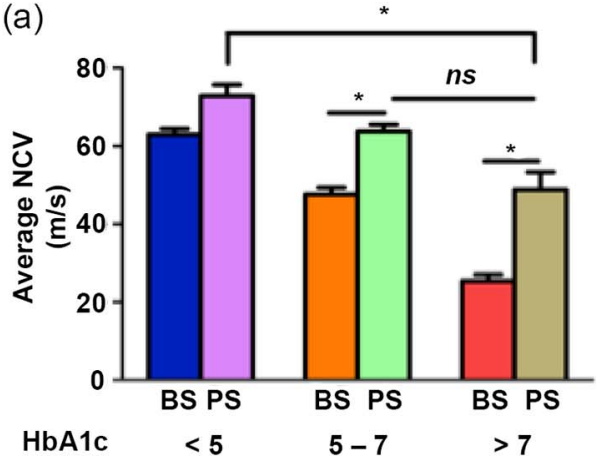

(c)

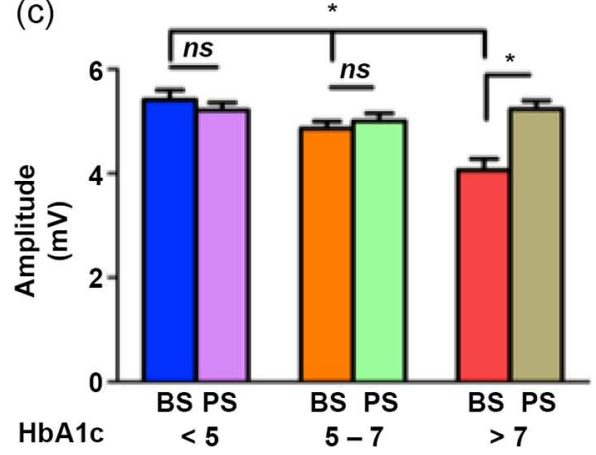

(b)

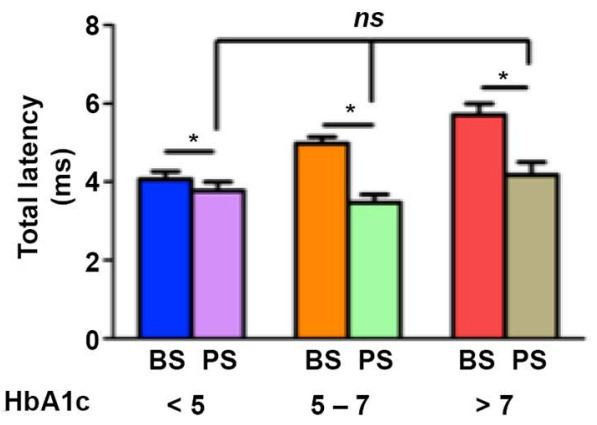

BS - Before surgery

PS - Post surgery

Fig. 2. Nerve conduction studies in the CTS individuals. Values expressed as mean $\pm \mathrm{SE}$ and $* p<0.05$ was considered as significant. (a) Nerve conduction velocity of the median nerve and resultant CMAP responses

following stimulation of the nerve at the wrist and elbow, showing significantly reduced nerve conduction prominently in the uncontrolled diabetic group. Restoration of the nerve conduction was observed in post-surgery and with control of the diabetes. (b and c) Comparison of standardized latencies and amplitude as recorded during motor nerve conduction studies on the median nerves. The diabetic group showed increased latency, which was restored after surgery (b). The amplitude was significantly low in the uncontrolled diabetic group compared with the other groups and post-surgical/diabetic control restored the amplitude (c) in the diabetic group

\section{Prognosis of CTS after surgery and restoration of the physical functions}

All the cases presented here were diagnosed according to the clinical and electrophysiological screening indicated elsewhere (Table III). The CTS post-surgical outcomes were evaluated for wound infection, scar tenderness, reflex sympathetic dystrophy, and finally

Table III. Relation of clinical severity and electrodiagnostic nerve muscle grading

\begin{tabular}{|c|c|c|c|c|}
\hline Classification of severity & $\begin{array}{c}\text { Electrophysiological } \\
\text { screening }\end{array}$ & $\mathbf{n = 6 4}$ & $\mathbf{\%}$ & $\begin{array}{c}\text { Mean Hi-Ob Scale }-\% \\
\text { severity score }\end{array}$ \\
\hline Mild & $\begin{array}{c}\text { Slowing of sensory nerve, } \\
\text { normal motor conduction }\end{array}$ & 6 & 10 & 3 \\
\hline Moderate & $\begin{array}{c}\text { Prolongation of motor and } \\
\text { sensory conduction }\end{array}$ & 20 & 31 & 3 \\
\hline Severe & $\begin{array}{c}\text { Sensory nerve action potential/ } \\
\text { CMAP absent }\end{array}$ & 38 & 59 & 4 \\
\hline
\end{tabular}


Table IV. Complications of CTS post-surgery and recovery

\begin{tabular}{|c|c|c|}
\hline Condition & Symptoms & Post-surgical recovery, \% \\
\hline \multirow{6}{*}{$\operatorname{HbA1c}<5(n=14)$} & Scar tenderness & 79 \\
\hline & Surgical site infection & 86 \\
\hline & Retained exposed stitch & 0 \\
\hline & Stiffness of the wrist and fingers & 86 \\
\hline & Hypertrophied scar & 93 \\
\hline & Residual numbness & 93 \\
\hline \multirow{6}{*}{$\mathrm{HbA} 1 \mathrm{c}=5-7(n=27)$} & Scar tenderness & 74 \\
\hline & Surgical site infection & 87 \\
\hline & Retained exposed stitch & 92 \\
\hline & Stiffness of the wrist and fingers & 91 \\
\hline & Hypertrophied scar & 96 \\
\hline & Residual numbness & 96 \\
\hline \multirow{6}{*}{$\mathrm{HbA} 1 \mathrm{c}>7(n=23)$} & Scar tenderness & 48 \\
\hline & Surgical site infection & 85 \\
\hline & Retained exposed stitch & 89 \\
\hline & Stiffness of the wrist and fingers & 93 \\
\hline & Hypertrophied scar & 93 \\
\hline & Residual numbness & 85 \\
\hline
\end{tabular}

the ability to do daily activities casually without the interference of any symptoms (Table IV). It was evident from our results that general surgical effects such as pain and paresthesia/numbness were reduced by $94 \%$ and $67 \%$, respectively, while $14 \%$ showed improvement in muscle weakness (Table I). Specifically in symptoms associated with CTS and post-operative recovery, $52 \%$ had improved scar tenderness out of $85 \%$ showing surgical site wound infection in Group III. In all the cases, irrespective of their diabetic profile, we did not observe any reflex sympathetic dystrophy (Table I). Interestingly, the severity in Groups II and III subsided within 3 weeks.

\section{Effect of $\left[\mathrm{Ca}^{2+}\right]_{c}$ in the vascular ECs and coherent high oxidative stress phenotype}

To check the oxidative burden of the cells adjoining the site of the CTS, we isolated ECs from some cases $(n=3)$ to assess the intracellular calcium levels and human umbilical vein endothelial cells that were used as control cells. The results showed a marked increase in the basal cytosolic calcium $\left[\mathrm{Ca}^{2+}\right]_{\mathrm{c}}$ compared with the ECs from the control (Fig. 3a). However, the high basal $\left[\mathrm{Ca}^{2+}\right]_{\mathrm{c}}$ observed in the initial passages became normal with increasing passages (Fig. 3b). We then measured cytosolic ROS (cROS) and found that the ECs isolated from Group III had increased cROS and our results very well corroborated with the increased basal $\left[\mathrm{Ca}^{2+}\right]_{\mathrm{c}}$ (Fig. 3c). The increased basal $\left[\mathrm{Ca}^{2+}\right]_{\mathrm{c}}$ and the corresponding cROS were also 
(a)

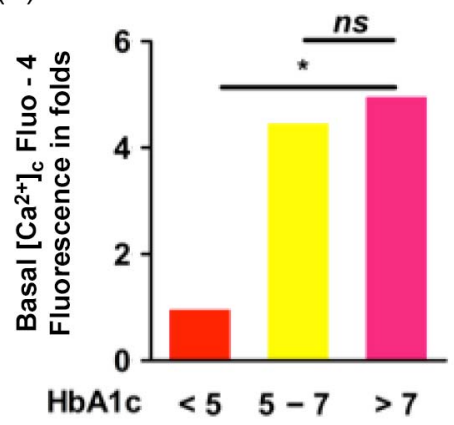

(b)

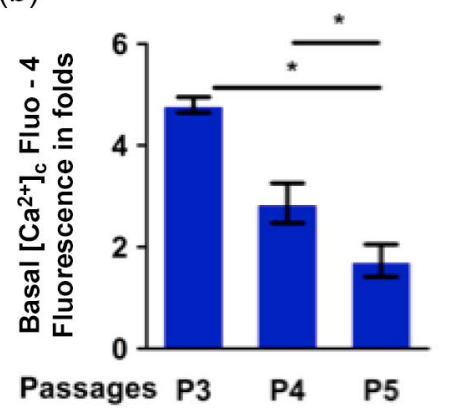

(c)

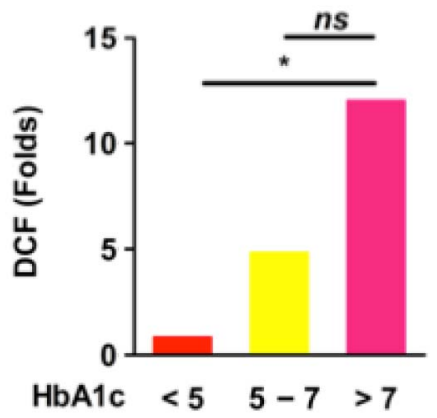

(d)

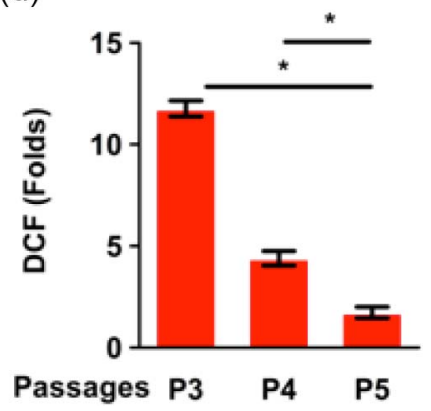

Fig. 3. Elevated basal $\mathrm{Ca}^{2+}$ associated endothelial manifestations. Data are mean $\pm \operatorname{SEM}(n=3 /$ group $) .{ }^{*} p<0.05$. (a) Basal cytosolic calcium $\left[\mathrm{Ca}^{2+}\right]_{\mathrm{c}}$ in the ECs isolated during the surgical procedure. Significantly increased $\left[\mathrm{Ca}^{2+}\right]_{\mathrm{c}}$ was observed in both diabetic groups at the initial passage levels, an indication of the stress phenotype as the resultant of the diabetes or CTS as such. (b and d) The late passage of the isolated ECs shows a rescue phenotype, where restoration of elevated basal cytosolic calcium $\left[\mathrm{Ca}^{2+}\right]_{\mathrm{c}}$ and elevated ROS to near-normal levels save the cells from high stress and subsequent cell death and indicate the restoration of the stress-free environment. The difference in the basal calcium and corresponding ROS is shown at passage 3, 4 and 5 levels, respectively. (c) Increased ROS was observed in the uncontrolled diabetic ECs than in controlled and non-diabetic cells. There was a progressive relationship with the elevated basal cytosolic calcium $\left[\mathrm{Ca}^{2+}\right]_{\mathrm{c}}$ and ROS

observed in Group I. However, the magnitude of the elevated values was significantly lower than that of the CTS diabetic phenotype observed in Groups II and III, whereas they were convincingly apparent when compared with the control cells (Fig. 3a and c). Interestingly, we observed restoration of the cROS to normalcy with corresponding fall in the $\left[\mathrm{Ca}^{2+}\right]_{\mathrm{c}}$ levels in the cells of higher passage numbers (Fig. 3d).

\section{Discussion}

Many studies have shown that diabetes or diabetic neuropathy may be a predisposing factor for CTS; however, a very few studies have attributed the role of the ECs resulting in microangiopathy to be an essential pathology of median nerve compression in CTS $(9,31)$. All the individuals underwent open release of flexor retinaculum by the orthopedic surgeon. The intricacies of the surgical procedure are beyond the scope of this study; therefore, they are not elaborated or discussed in this manuscript. All the 64 individuals diagnosed with CTS 
by clinical examination and electrodiagnostic studies, irrespective of diabetic status, were considered for surgery as the symptoms were interfering with their daily activities (Table I). The average age at surgery was above 44 in both males and females; however, the females outnumbered males by more than $50 \%$. This was in agreement with the previously published studies in the literature (32). Repetitive wrist motion was implicated in the pathogenesis of CTS (26). In the current investigations, we observed that the T2DM delayed the post-surgical prognosis of CTS, and a possible reason could be the glycosylation of the proteins of the tendons, thereby triggering worsening inflammation with exacerbation by repeated forceful flexing of the wrists (28). It is more complex to diagnose CTS in the diabetic cases, as tingling, prickling, or numbness due to peripheral neuropathy may co-present, which is again unrelated to CTS and may be a prominent factor undermining the CTS itself $(6,25)$. In this study, about $77 \%$ of individuals experienced pain with $87 \%$ showing numbness in the distribution of the median nerve. Nearly $66 \%$ of individuals showed muscle weakness, which is in good agreement with the above argument of diabetic cases more often going undiagnosed for CTS. It may be noted that we did not include Tinel's and Phalen's signs as these are not considered essential for the diagnosis or selecting the cases due to their profound false positivity (10). Involvement of both the hands (bilateral) was least observed, as most of the individuals showed CTS in the right hand. This observation may be the tip of an iceberg regarding occupational hazards, as inclusion of a greater number of cases and individuals based on mode of work or profession can throw light on hand involvements. The results (Fig. 1c) showing predominant right-hand involvement were not in agreement with the previous studies (33).

In post-surgery, the individuals were divided into groups based on their diabetic HbA1c levels, so that the impact of T2DM on CTS prognosis with respect to clinical symptoms can be analyzed. The results were discriminatory, although there was a brief period of glycemic control pre- and post-surgery, especially in Group III. The altered NCV and the distal motor latency were low and more prolonged, respectively, in Group III compared with Groups I and II. We further observed low amplitude, but only in Group III. We did not observe any significant changes pertaining to CTS symptoms like reflex sympathetic dystrophy (data not shown) due to altered nerve conduction studies increasingly worsening with the diabetic status. Interestingly, histology reveals thickening of the extracellular matrix is characterized with actively proliferating endothelial, smooth muscle, and synovial lining cells with higher expression of VEGF and other cellular factors (7). Our investigations showed a direct relationship of the nerve conduction studies with diabetic status and oxidative burden of the cells from CTS. However, our study population in each group was not sufficiently numerous to arrive at a significant conclusion on this issue. This part of our work should stimulate a greater study in depth of these nerve conduction parameters in the larger group of individuals with CTS. However, as expected, the HbA1c levels were well corroborated with the $\mathrm{NCV}$, latency, and amplitude and were in agreement with other observations (22).

The role of intracellular calcium levels was implicated with pathology as early as 1980 with a few investigations highlighting the increased free cytosolic calcium as damaging cellular factor $(7,11,29)$. Through our publications, we have previously shown the implications of $\left[\mathrm{Ca}^{2+}\right]_{\mathrm{c}}$, and thereby cROS in cellular models leading to cell death and induced apoptosis. High external pressure (environmental stress) due to compression, as observed in CTS, may be attributed to the elevated $\left[\mathrm{Ca}^{2+}\right]_{\mathrm{c}}$ and high ROS mediated oxidative state, thereby skewing the cells toward death and worsening the pathology. 
Alteration in the redox state of the cells may induce the release of the $\mathrm{Ca}^{2+}$ from the endoplasmic reticulum into the cytosol, adding to the already high $\mathrm{Ca}^{2+}$ observed due to external flooding through plasma membrane channels, resulting in cells to undergo apoptosis or necrosis $(17,19)$. The mechanism behind the stress, which is observed in CTS as well as in diabetic neuropathy, cannot be fully delineated and the role of mitochondrial $\mathrm{Ca}^{2+}$ and ROS needs to be properly addressed. In our previous studies, we have shown the relationships between the elevated $\left[\mathrm{Ca}^{2+}\right]_{\mathrm{c}}$ and $\left[\mathrm{Ca}^{2+}\right]_{\mathrm{m}}$ that result in activation of the cells through a mitochondria-dependent, apoptotic pathway (18). However, whether apoptosis of ECs occurs due to the stress induced by the CTS or by the diabetic neuropathy, or a combination of these processes, needs to be further investigated at the level of transcriptional regulation. In this study, we clearly show the intervention of CTS and control of the diabetes reduces the associated endothelial pathology, even in the nondiabetic CTS cases. We were further satisfied with our results regarding the elevated basal $\left[\mathrm{Ca}^{2+}\right]_{\mathrm{c}}$, and thereby high cROS can be implicated in the extensive lower motor and sensory nerve conduction velocities classically observed in CTS with T2DM complicating the clinical severity as well as the post-surgical outcome. Since our investigation was focused on the endothelial activation and dysfunction, our criteria for selection of the patients were stringent on the scale of $\mathrm{HbA} 1 \mathrm{c}$ levels and not by changes in nerve conduction studies. We tried to correlate our post-surgical prognosis with oxidative stress of ECs. These ECs are known to be the first line of cells to encounter stress, which can be exerted physiologically through compression or persistent high plasma glucose (as in our case) to be the reason for higher $\left[\mathrm{Ca}^{2+}\right]_{\mathrm{c}}$ and thereby ROS. Some of the studies on oxidative stress in CTS have shown the involvement of activated endothelial NF- $\mathrm{KB}$ along with the higher expressions of eNOS and TGF- $\beta$ (16). Interestingly, we observed that elevation of $\mathrm{Ca}^{2+}$ correlated well with the diabetic status of the CTS individuals with fluctuating glycemic controls in Groups II and III aiding in worsening the clinical picture of CTS.

\section{Conclusion}

From the overall results, we observed that the control of T2DM in CTS will result in better post-surgical outcome. Long-term control of the diabetes is the most favored approach with a recommendation for screening for diabetes in all cases of referred CTS. We observed a significant correlation of nerve conduction studies with the clinical severity, which reinforced the diabetic role in the pathology of CTS. Furthermore, the results of the nerve conduction studies clearly corroborated the $\left[\mathrm{Ca}^{2+}\right]_{c}$ and cROS values observed in the ECs isolated from the individuals with CTS. Together, these results show that T2DM predisposing the cells to more stress via $\mathrm{Ca}^{2+}$ and ROS not only worsens the clinical severity of CTS but also prolongs the healing process following surgery. Based on these results, one could suggest a trial of conservative management for individuals with CTS.

\section{Acknowledgements}

The authors would like to thank Dr. Rajeev Rao, Dr. Sujith V.C, and Dr. Girish from the Department of Orthopedics and Dr. Gilvaz, Consultant Neurologist, Jubilee Mission Medical College, Thrissur, Kerala, India for their help and valuable suggestions throughout the work. The authors would also like to thank Mr. Mahmoud Al-Khateeb, Department of Physiology, College of Medicine, King Khalid University, Abha, Saudi Arabia for his help in some of the experiments. 


\section{REFERENCES}

1. Basiri K, Katirji B: Practical approach to electrodiagnosis of the carpal tunnel syndrome: a review. Adv. Biomed. Res. 4, 50 (2015)

2. Bin-Jaliah IM, Shariff ME, Chandramoorthy HC: Elevated basal cytosolic calcium influences the postsurgical outcome in diabetic patients with carpal tunnel syndrome. Proceedings of the 38th Annual meeting of the Japan Neuroscience Society, Kobe, Japan, 26-31 July 2015

3. Borradaile NM, Han X, Harp JD, Gale SE, Ory DS, Schaffer JE: Disruption of endoplasmic reticulum structure and integrity in lipotoxic cell death. J. Lipid Res. 47, 2726-2737 (2006)

4. Chammas M, Boretto J, Burmann LM, Ramos RM, Dos Santos Neto FC, Silva JB: Carpal tunnel syndrome part I (anatomy, physiology, etiology and diagnosis). Rev. Bras. Ortop. 49, 429-436 (2014)

5. Deger AN, Deger H, Taser F: The role of neoangiogenesis and vascular endothelial growth factor in the development of carpal tunnel syndrome in patients with diabetes. Niger. J. Clin. Pract. 19, 189-195 (2016)

6. Diabetic Neuropathies: The nerve damage of diabetes. National Institute of Diabetes and Digestive and Kidney Diseases website. Available at: http://www.niddk.nih.gov/health-information/health-topics/Diabetes/diabeticneuropathies-nerve-damage-diabetes/Pages/diabetic-neuropathies-nerve-damage.aspx. Updated 26 Nov 2013.

7. Hagberg M (1987): Shoulder pain pathogenesis. In: Clinical Concepts in Regional Musculoskeletal Illness, ed Hadler NM, Grune and Stratton, Orlando, FL, USA, pp. 191-200

8. Haidara MA, Assiri AS, Youssef MA, Mahmoud MM, Eajaz A, Al-Hakami A, Chandramoorthy HC: Differentiated mesenchymal stem cells ameliorate cardiovascular complications in diabetic rats. Cell Tissue Res. 359, 565-575 (2015)

9. Hirata H, Nagakura T, Tsujii M, Morita A, Fujisawa K, Uchida A: The relationship of VEGF and PGE2 expression to extracellular matrix remodelling of the tenosynovium in the carpal tunnel syndrome. J. Pathol. 204, 605-612 (2004)

10. Ibrahim I, Khan W, Goddard N, Smitham P: Carpal tunnel syndrome: a review of the recent literature. Open Orthop. J. 6, 69-76 (2012)

11. Imbriglia JE, Boland DM: An exercise-induced compartment syndrome of the dorsal forearm - a case report. J. Hand Surg. Am. 9, 142-143 (1984)

12. Jablecki CK, Andary MT, Floeter MK, Miller RG, Quartly CA, Vennix MJ, Wilson JR: Electrodiagnostic studies in carpal tunnel syndrome. Neurology 58, 1589-1592 (2002)

13. Jeppsson JO, Kobold U, Barr J, Finke A, Hoelzel W, Hoshino T, Miedema K, Mosca A, Mauri P, Paroni R, Thienpont L, Umemoto M, Weykamp C: Approved IFCC reference method for the measurement of $\mathrm{HbA} 1 \mathrm{c}$ in human blood. Clin. Chem. Lab. Med. 40, 78-89 (2002)

14. Jinrok O, Zhao C, Amadio PC, An KN, Zobitz ME, Wold LE: Vascular pathologic changes in the flexor tenosynovium (subsynovial connective tissue) in idiopathic carpal tunnel syndrome. J. Orthop. Res. 22, 13101315 (2004)

15. Kayali H, Kahraman S, Sirin S, Bedük A, Timurkaynak E: Bilateral carpal tunnel syndrome with type 1 diabetes mellitus in childhood. Pediatr. Neurosurg. 38, 262-264 (2003)

16. Kim JK, Koh YD, Kim JS, Hann HJ, Kim MJ: Oxidative stress in subsynovial connective tissue of idiopathic carpal tunnel syndrome. J. Orthop. Res. 28, 1463-1468 (2010)

17. Li L, Tan H, Gu Z: Heat stress induces apoptosis through a $\mathrm{Ca}^{2+}$-mediated mitochondrial apoptotic pathway in human umbilical vein endothelial cells. PLoS One 9, 1-16 (2014)

18. Mallilankaraman K, Cárdenas C, Doonan PJ, Chandramoorthy HC, Irrinki KM, Golenár T, Csordás G, Madireddi P, Yang J, Müller M, Miller R, Kolesar JE, Molgó J, Kaufman B, Hajnóczky G, Foskett JK, Madesh M: MCUR1 is an essential component of mitochondrial $\mathrm{Ca}^{2+}$ uptake that regulates cellular metabolism. Nat. Cell Biol. 14, 1336-1343 (2012)

19. Mallilankaraman K, Doonan P, Cárdenas C, Chandramoorthy HC, Müller M, Miller R, Hoffman NE, Gandhirajan RK, Molgó J, Birnbaum MJ, Rothberg BS, Mak DO, Foskett JK, Madesh M: MICU1 is an essential gatekeeper for MCU-mediated mitochondrial $\mathrm{Ca}^{2+}$ uptake that regulates cell survival. Cell 151, 630644 (2012)

20. Moghtaderi A, Dabiri S, Dahmardeh M: Asymptomatic carpal tunnel syndrome in obese and overweight patients with metabolic syndrome. Neurosciences (Riyadh) 18, 87-90 (2013)

21. Mojaddidi MA, Ahmed MS, Ali R, Jeziorska M, Al-Sunni A, Thomsen NO, Dahlin LB, Malik RA: Molecular and pathological studies in the posterior interosseous nerve of diabetic and non-diabetic patients with carpal tunnel syndrome. Diabetologia 57, 1711-1719 (2014) 
22. Neha HP, Kinnar SD, Amita KM, Toral MG, Mitesh NS, Vaishali NP: Electrophysiological changes in sensorimotor nerves in diabetes mellitus \& usefulness of nerve conduction studies for early diagnosis of diabetic neuropathy. Int. J. Biomed. Adv. Res. 4, 187-191 (2013)

23. Nourbakhsh MR, Bell TJ, Martin JB, Arab AM: The effects of oscillatory biofield therapy on pain and functional limitations associated with carpal tunnel syndrome: randomized, placebo-controlled, double-blind study. J. Altern. Complement. Med. 22, 911-920 (2016)

24. Paryavi E, Zimmerman RM, Means KR Jr: Endoscopic compared with open operative treatment of carpal tunnel syndrome. JBJS Rev. 4, e2 (2016)

25. Pourmemari MH, Shiri R: Diabetes as a risk factor for carpal tunnel syndrome: systematic review and metaanalysis. Diabet. Med. 33, 10-16 (2016)

26. Reale F, Ginanneschi F, Sicurelli F, Mondelli M: Protocol of outcome evaluation for surgical release of carpal tunnel syndrome. Neurosurgery 53, 343-350 (2003)

27. Saint-Lary O, Rébois A, Mediouni Z, Descatha A: Carpal tunnel syndrome: primary care and occupational factors. Front. Med. (Lausanne) 2, 28 (2015)

28. Singh VP, Bali A, Singh N, Jaggi AS: Advanced glycation end products and diabetic complications. Korean J. Physiol. Pharmacol. 18, 1-14 (2014)

29. Sjøgaard G: Exercise-induced muscle fatigue: the significance of potassium. Acta Physiol. Scand. Suppl. 593, 1-63 (1990)

30. Sud V, Freeland AE: Biochemistry of carpal tunnel syndrome. Microsurgery 25, 44-46 (2005)

31. Vinik A, Mehrabyan A, Colen L, Boulton A: Focal entrapment neuropathies in diabetes. Diabetes Care 27, 1783-1788 (2004)

32. Weber RA, Rude MJ: Clinical outcomes of carpal tunnel release in patients 65 and older. J. Hand Surg. Am. 30, 75-80 (2005)

33. Zhang W, Johnston JA, Ross MA, Smith AA, Coakley BJ, Gleason EA, Dueck AC, Santello M: Effects of carpal tunnel syndrome on adaptation of multi-digit forces to object weight for whole-hand manipulation. PLoS One 6, e27715 (2011) 